\title{
Subcutaneous versus Sublingual Immunotherapy for Allergic Rhinitis therapy: Which Is Superior
}

\author{
Magdy Abdullah Sayedelahl ${ }^{1}$, Naser Nagib Nasr ${ }^{1}$, Mahmoud Hamed Akr ${ }^{1}$, Dina Sayed Sheha ${ }^{2, *}$, \\ Tahany Mohamed Rabie ${ }^{3}$
}

${ }^{1}$ Department of Otorhinolaryngology, Faculty of Medicine, Zagazig University, Zagazig, Egypt

${ }^{2}$ Department of Internal Medicine, Allergy and Clinical Immunology, Faculty of Medicine, Ain Shams University, Cairo, Egypt

${ }^{3}$ Department of Otorhinolaryngology, Faculty of Medicine, Ain Shams University, Cairo, Egypt

\section{Email address:}

mega1111958@gmail.com (M. A. Sayedelahl),ent.laser@yahoo.com (N. N. Nasr),mahmoud0114@yahoo.com (M. H. Akr), shehadina@yahoo.com (D. S. Sheha), tahany.rabie@gmail.com (T. M. Rabie)

\section{To cite this article:}

Magdy Abdullah Sayedelahl, Naser Nagib Nasr, Mahmoud Hamed Akr, Dina Sayed Sheha, Tahany Mohamed Rabie. Subcutaneous versus Sublingual Immunotherapy for Allergic Rhinitis therapy: Which Is Superior. International Journal of Immunology.

Vol. 3, No. 3, 2015, pp. 42-46. doi: 10.11648/j.iji.20150303.13

\begin{abstract}
Background: A randomized single-blinded study including 50 patients with allergic rhinitis. Objective: To evaluate and compare the efficacy of subcutaneous versus sublingual immunotherapy in treatment of allergic rhinitis. Materials and methods: Patients divided into Group A: twenty patients received subcutaneous immunotherapy and group B: twenty patients received sublingual immunotherapy for twelve months. We assessed skin prick test, symptom score and medication use, quality of life and nasal smear eosinophilic count before and after treatment. Results: In group A, clinical improvement was achieved in $100 \%$ of monosensitised and $62.5 \%$ of polysensitised patients, while in group B $100 \%$ of monosensitised and $60 \%$ of of polysensitised patients exhibited clinical improvement. Conclusion: The subcutaneous and sublingual routes of immunotherapy have similar efficacy.
\end{abstract}

Keywords: Allergen Specific Immunotherapy, SCIT, SLIT, Allergic Rhinitis, Skin Prick Test, Nasal Smear Eosinophilia, Clinical Improvement

\section{Introduction}

Allergic rhinitis (AR) is an IgE-mediated inflammation of the upper airway that involves inflammation of the mucous membranes of the nose, eyes, Eustachian tubes, middle ear, sinuses, and pharynx [1]. Immunotherapy, both subcutaneous (SCIT) and sublingual (SLIT), is an effective treatment for adults and children with severe AR not responding to allergen avoidance measures and conventional pharmacotherapy [2]. Although AR is the disease in which SCIT efficacy is most documented and proved [3], constraints of the injective route and possibility of severe systemic reactions led to continuous research for alternative routes (nasal, oral, bronchial and sublingual), with the sublingual route generating most interest [4]. The clinical effect of both modalities lasts at least 3 years after stopping treatment [5].

The main aim of our study was to evaluate and compare the long term clinical efficacy of SCIT versus SLIT in treatment of patients with AR.

\section{Materials and Methods}

Our study was a randomized single-blinded study including 50 patients with moderate to severe AR recruited from Allergy outpatient clinic at Ain Shams and Zagazig University Hospitals from May 2013 till September 2014. The study was approved by the local ethics committee and all patients gave informed oral consent. Diagnosis of AR conformed to ARIA Guidelines 2010 revision [6].

Table 1. Allergen extracts included in skin prick test.

\begin{tabular}{lll}
\hline Mixed molds & Mixed pollen & House dust \\
\hline Mite & Wool & Tobacco \\
Candida & Latex & Goat hair \\
Hay dust & Feather & Pigeon \\
Cat hair & Cotton dust & Dog hair \\
Cockroaches & & \\
\hline
\end{tabular}


Skin prick test (SPT) was done to identify allergen/allergens for which they will receive immunotherapy We included patients with a positive SPT to 1 up to a maximum of 3 of the commonest locally encountered aeroallergens. The test panel was prepared at the Allergy and Clinical Immunology Unit laboratory at Ain Shams University hospitals (table 1).

Exclusion criteria included patients with positive SPT to more than 3 aeroallergens, inflammatory or septic conditions, patient with end organ failure, patients who received steroids, cytotoxic drugs or immunosuppressives one month prior to sample collection.

Of the 50 patients, 5 withdrew from each group due to poor adherence to treatment, relocation and pregnancy. Remaining patients were divided into two groups.

Group A: 20 patients received SCIT and group B: 20 patients received SLIT.

Both groups received immunotherapy for 12 months. The items assessed before and after the duration of treatment included SPT, patient symptom score, medication use, quality of life and nasal smear eosinophilic count.

\subsection{Skin Prick Test (SPT)}

SPT was performed on healthy skin on the volar surface of the forearm at $3-\mathrm{cm}$ intervals. A lancet (Dome-Hollister-Stier) was used to prick the skin at a $90^{\circ}$ angle through a drop of test solution on the skin [7]. A drop of histamine phosphate and a drop of saline $0.9 \%$ were used as a positive control and negative control respectively. The immediate response was evaluated after 20 minutes. A wheal diameter $\geq 3 \mathrm{~mm}$ more than the negative control was considered to be positive [8].

\subsection{Quality of Life}

The Mini Rhinoconjunctivitis Quality of Life Questionnaire (MiniRQLQ) was used, comprised of 14questions (items) in five domains (activity limitations, sleep impairment, practical problems, nasal symptoms, eye symptoms and emotional problems) [9].

\subsection{Medication Use}

Medication usage was scored on a 4-point scale (table 2).

Table 2. Medication score.

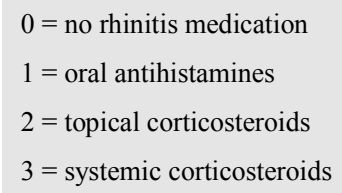

Patients were considered improved if the total score decreased by one or more points [10].

\subsection{Nasal Smear Eosinophilia}

Anterior rhinoscopy was performed after application of a local vasoconstrictor (xylometazoline $0.1 \%$ ). With a cotton swab, a smear was taken from the posterior part of the inferior or middle turbinate. The secretions were spread out to a thin layer on a glass slide and air-dried. Later, the smear was stained by the May-Griinwald- Giemsa method for microscopic evaluation [11].

\subsection{Allergen-specific Immunotherapy (SIT)}

A) Subcutaneous immunotherapy (SCIT):

Extracts were prepared as an aqueous solution using the weight/volume (wt/vol) method. A potency of 1:100 indicates that $1 \mathrm{~g}$ of dry allergen was added to $100 \mathrm{cc}$ of a buffer (phenol saline) for extraction. The allergen was eluted for a time, and then the solid material was filtered out, leaving an aqueous solution [12].

Injections were administered with a 1-ml syringe. The injections were administered subcutaneously in the posterior portion of the middle third of the upper arm [13].

Dose: Allergen extract was diluted to have final dilutions $1: 100,1: 1000$ and $1: 10000$. The starting point was $0.2 \mathrm{ml}$ of a 1:10000 dilution. The injections were twice weekly and the dose was increased weekly: $0.2,0.4,0.6,0.8$ and $1 \mathrm{ml}$. The procedure was repeated with the next higher concentrations until the maintenance dose was reached.

Premedication: Patients were prescribed oral antihistamines 2 hours before each injection to reduce the frequency of any systemic reactions [13].

B) Sublingual immunotherapy (SLIT):

Extracts were prepared as glycerinated solution, using the wt/vol method [14]. Extract was administered on an empty stomach as sublingual drops kept under the tongue for 2 minutes then swallowed.

Dose: Treatment was divided into build-up and maintenance phases. Allergen extract was diluted to have final dilutions 1:50, 1:500 and 1:5000. The starting dose was 3 drops daily of 1:5000 dilution for one week, then 5 drops daily for the second week, then 7 drops daily for another third week. The procedure was repeated with the next higher concentrations until the maintenance dose was reached, which was 5 drops of 1:50 dilution. The maintenance dose was given every other day for 3 weeks then every 3 days for 6 weeks then every week for a total of 12 months.

Statistical Analysis:

Analysis of data was performed on a personal computer using Graph Pad Prism version 5, as follows:

1. Description of quantitative variables using mean, standard deviation (SD) and range.

2. Description of qualitative variables using number and percentage.

3. Unpaired t-test was used to determined significant differences between two different groups.

4. Paired t-test was used to determined significant differences within the same group.

5. The level of significance was $(\mathrm{p}<0.05)$.

\section{Results}

Baseline demographic and clinical characteristics are displayed in table 3.Regarding SPT in group A, 4 patients 
(20\%) were monosensitized, 2 patients for house dust mites, 1 for pollens, 1 for pigeon feather and 16 patients were polysensitized. For group B, 5 patients (25\%) were monosensitized, 3 patients for pollens, 1 for hay dust, 1 for house dust mites and 15 patients were polysensitized.

Regarding SPT, patients were considered improved if the test became negative or number of positive allergens diminished after 12 months of immunotherapy, while persistence of the same allergens or appearance of new allergens denoted that the patient didn't improve (fig 1).Table 4 shows number of patients who showed clinical improvement in both groups. Although there was statistically significant improvement in both the SCIT group and SLIT group after administering immunotherapy, we could not detect statistically significant difference between both groups after treatment indicating equal efficacy of both SCIT and SLIT in causing clinical improvement of allergic rhinitis patients after 12 months of SIT. Single-allergen preparations were clearly more effective than multi-allergen preparations in control of symptoms and improvement of the patient in both studied groups. As illustrated in table 4 Fig. (2), comparing the pre and post treatment medication use mean values were $1.950 \pm 0.223$ and $1.250 \pm 0.638$ for group A (SCIT) and $1.950 \pm 0.223$ and $1.33 \pm 0.656$ for group B (SLIT) which indicated statistically significant difference in each group between the pre and post treatment $(p<0.05)$. As illustrated in Fig. (3), when comparing the pre and post treatment nasal smear eosinophilia mean values were $7.65 \pm 3.06$ and $5.20 \pm 3.31$ for SCIT group and $8.8 \pm$ 2.33 and $4.9 \pm 3.024$ for SLIT group indicating statistically significant difference in both groups post treatment $(\mathrm{p}<0.05)$.

Regarding side effects noted, in SCIT group 13 patients experienced local redness and edema at the injection site, 1 patient had overall itching, 2 patients experienced acute asthmatic attacks. In SLIT group, 1 patient complained of diarrhea and abdominal pain, 1 patient had oral itching, and 1 patient had an asthmatic attack.

Table 3. Baseline demographic and clinical characteristics of the 2 groups.

\begin{tabular}{lll}
\hline Variable & Group A(n=20) & Group B (n=20) \\
\hline Age, year & $36.8(9.277)$ & $29.3(11.75)$ \\
Male/Female ratio & $8 / 12$ & $15 / 5$ \\
Medication score & $1.950(0.233)$ & $1.950(0.233)$ \\
Baseline mini RQLQ & $43.30(8.64)$ & $35.55(9.67)$ \\
Nasal smear eosinophilia (\%) & $7.65(3.06)$ & $8.8(2.33)$ \\
\hline
\end{tabular}

Data presented as mean (SD) or number (\%)

Table 4. Number (\%) of patients who showed clinical improvement (mini $R Q L Q)$ in both groups.

\begin{tabular}{lll}
\hline Item & $\begin{array}{l}\text { Monosensetized } \\
\text { patients }\end{array}$ & $\begin{array}{l}\text { Polysensitized } \\
\text { patients }\end{array}$ \\
\hline No of improved patients Group A & $(4) 100 \%$ & $(10) 62.5 \%$ \\
No of improved patients Group B & $(5) 100 \%$ & $(9) 60 \%$ \\
\hline
\end{tabular}

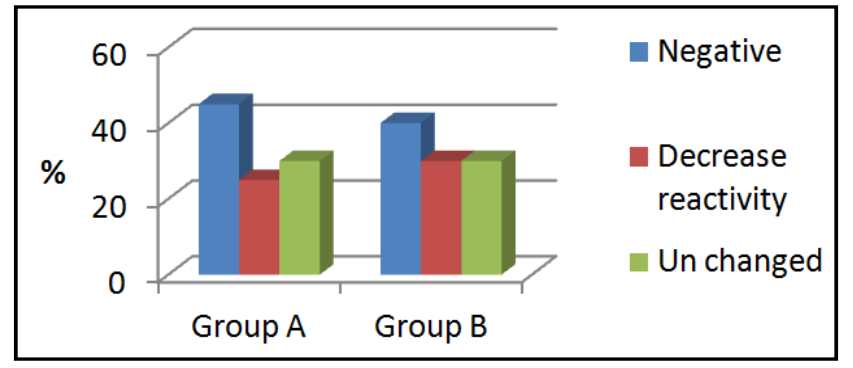

Fig. (1). The Skin prick test in both group A (SCIT) and group B (SLIT) after 12 months of treatment.

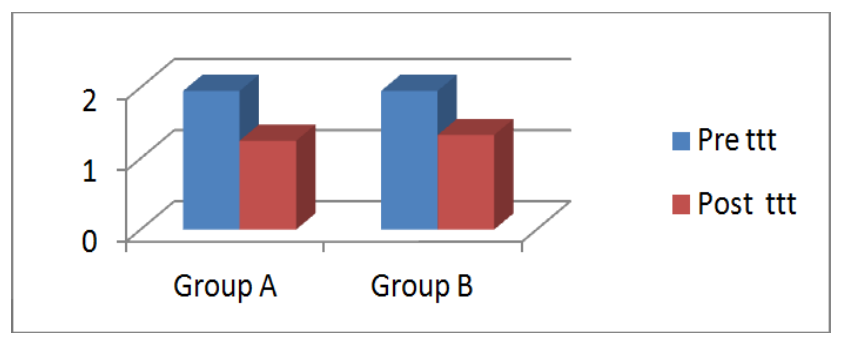

Fig. (2). Medication score of each SCIT and SLIT group before and after treatment.

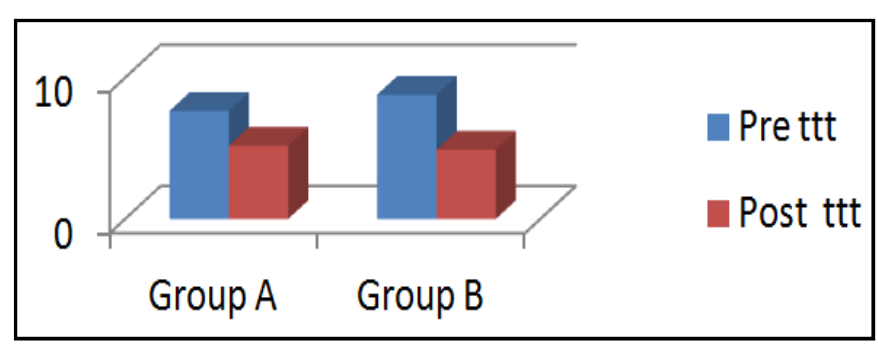

Fig. (3). Nasal smears eosinophilia of each SCIT and SLIT group before and after treatment.

\section{Discussion}

AR represents a major cause of morbidity that includes interference with usual daily activities and impairment of sleep quality [15]. Immunotherapy can be administered by different routes; the classical SCIT usually known as "allergy shots." and SLIT where the allergens are administered as drops to the sublingual area [16]. Side effects reported after immunotherapy in our study were more pronounced in the SCIT rather than SLIT group.

In both groups we found clinical improvement after 12 months of treatment, with marked reduction in medication use; 4 patients stopped their medications completely.

A systematic review done concluded there was significant reduction of symptom and medication scores following SLIT, however all studies included in the meta analysis administered SLIT in comparison to placebo rather than SCIT [17].

In 15 studies published between 2000 and 2006, reduction of symptoms and/or need for medications were confirmed with grass, birch, parietaria, ragweed pollens and house dust mites in SCIT treated patients. However, all of the studies included in the meta-analysis compared the efficacy of SCIT to placebo [18]. 
Another Cochrane meta-analysis of 42 double-blind, placebo-controlled studies showed significant reduction in rhinitis symptoms and medication requirements after SLIT [19].

Kim and Colleagues compared multiallergen SCIT in polysensitized patients with single-allergen SCIT in monosensitized patients. The study investigated 130 children treated for 18 months. In both groups the mean postallergen immunotherapy symptom scores were significantly $(\mathrm{P}<0.05)$ lower than preallergen immunotherapy. However, the reduction was significantly less intense in the polysensitized group [20].

Reduced skin test reactivity after immunotherapy was observed in both groups; this may be explained by the fact that SIT leads to an increase of allergen-specific IgG, especially IgG4, which leads to inhibition of IgE-facilitated antigen presentation and inhibition of IgE-mediated release of mediators from mast cells and basophils [21].

Similarly, Eifan and Colleagues also found that SPT response significantly decreased in both SLIT and SCIT groups compared to baseline [22]. Nasal smear eosinophil counts in both groups of this study exhibited a significant reduction after therapy, although not reaching statistical significance.

SIT was found to decrease the recruitment of mast cells, basophils, and eosinophils to the skin, nose, eye, and bronchial mucosa after exposure to allergens [23].

To our knowledge, only a few studies have directly compared SCIT and SLIT [3]. Ours is one of the very few studies to directly compare SCIT and SLIT regarding both clinical and immunologic parameters.In 2004, the first study comparing efficacy of SLIT versus SCIT in AR is yet considered as the only adequate methodological trial (placebo controlled, double blind, double dummy) [24], concluding that both SCIT and SLIT are clinically effective compared with placebo in treatment of birch pollen rhinoconjunctivitis.

We report comparable efficacy of SCIT and SLIT for AR treatment, with improvement in symptom control and reduced medication use. SLIT represents a more suitable alternative to SCIT suitable for home use and with a better safety profile and less adverse effects. Single allergen immunotherapy was more effective than multi-allergen immunotherapy. Since the indications of both types of immunotherapy are similar, the patient's choice is crucial in deciding whether to use the subcutaneous or sublingual route. There remains a need for larger double-blinded controlled long-term studies on immunotherapy with perennial allergens.

\section{Acknowledgment}

The authors declare no funding was received for the study, and they have no conflict of interest.

\section{References}

[1] Tahamiler R, Saritzali G, Canakcioglu S, Ozcora E, Dirican A. Comparison of the long-term efficacy of subcutaneous and sublingual immunotherapies in perennial rhinitis. ORL J Otorhinolaryngol Relat Spec. 2008; 70(3) 144-150.

[2] Walker, S. M., Durham, S. R., Till, S. J., Roberts, G., Corrigan, C. J., Leech, S. C., Krishna, M. T et al. Immunotherapy for allergic rhinitis. Clinical \& Experimental Allergy 2011; 41: $1177-1200$

[3] Pipet A, Botturi K, Pinot D, Vervloet D, Magnan A. Allergenspecific immunotherapy in allergic rhinitis and asthma. Mechanisms and proof of efficacy. Respir Med. 2009; 103:800-812.

[4] Canonica GW, Passalacqua G. Non injection routes for immunotherapy. J Allergy Clin Immunol 2003: 111: 437-48.

[5] Antu' nez C, Mayorga C, Corzo JL, Jurado A, Torres MJ. Two year follow-up of immunological response in mite-allergic children treated with sublingual immunotherapy. Comparison with subcutaneous administration. Pediatr Allergy Immunol 2008: 19: 210-218.

[6] Brozek JL, Bousquet J, Baena-Cagnani CE, Bonini S, Canonica GW, Casale TB et al. Allergic Rhinitis and its Impact on Asthma (ARIA) guidelines: 2010 revision. J Allergy Clin Immunol. 2010; 126:466-476.

[7] Dreborg S. Histamine reactivity of the skin. Allergy 2001; 56:359-64.

[8] Kapp A, Demarteau N. Cost effectiveness of levocetirizine in chronic idiopathic urticaria: A pooled analysis of two randomised controlled trials. Clin Drug Investig. 2006; 26:1-11.

[9] Juniper EF, Thompson AK, Ferrie PJ, Roberts JN. Development and validation of the mini Rhinoconjunctivitis Quality of Life Questionnaire ClinExp Allergy 2000;30:132140

[10] Canonica GW, Baena-Cagnani CE, Bousquet J, Bousquet PJ, Lockey RF, Malling HJ et al. Recommendations for standardization of clinical trials with Allergen Specific Immunotherapy for respiratory allergy. Allergy 2007; 62:317324.

[11] Crobach M, Hermans J, Kaptein A, Kaptein A, Ridderikhoff J, Mulder J. Nasal smear eosinophilia for the diagnosis of allergic rhinitis and eosinophilic non-allergic rhinitis. Scand $\mathbf{J}$ Prim Health Care 1996;14:116-121

[12] Joint Task Force on Practice Parameters. Allergen immunotherapy: a practice parameter. American Academy of Allergy, Asthma and Immunology. American College of Allergy, Asthma and Immunology. Ann Allergy Asthma Immunol. 2003; 90:1-40.

[13] Cox L, Li JT, Nelson H, et al. Allergen immunotherapy: A practice parameter - second update. J Allergy Clin Immunol 2007; 120: S25-85

[14] Marogna M, Spadolini I, Massolo A, Canonica GW, Passalacqua G. Clinical, functional, and immunologic effects of sublingual immunotherapy in birch pollinosis: A 3-year randomized controlled study. J Allergy Clin Immunol 2005; 115: 1184-8. 
[15] Dykewicz MS, Hamilos DL. Rhinitis and sinusitis. J Allergy Clin Immunol 2010; 125:S103-115

[16] Kleine-Tebbe J, Ribel M, Herold D. Safety of a standardised grass allergen tablet for sublingual immunotherapy a randomized, placebo-controlled trial," Allergy 2006; 61(2): $181-184$.

[17] Wilson DR, Lima MT, Durham SR. Sublingual immunotherapy for allergic rhinitis: systematic review and meta-analysis. Allergy 2005; 60:4-12.

[18] Passalacqua G, Durham SR. Allergic Rhinitis and its Impact on Asthma update: Allergen immunotherapy. Journal of Allergy and Clinical Immunology 2007;119 (4):881 - 891

[19] Radulovic S, Calderon MA, Wilson D, Durham S. Sublingual immunotherapy for allergic rhinitis. Cochrane Database Syst Rev 2010; 12:CD002893.

[20] Kim KW, Kim EA, Kwon BC, Kim ES, Song TW, Sohn MH et al. Comparison of allergic indices in monosensitized and polysensitised patients with childhood asthma. J Korean Med Sci 2006; 21:1012-6.

[21] Wachholz PA, Durham SR. Mechanisms of immunotherapy: IgG revisited. Curr Opin Allergy Clin Immunol 2004 4(4):313-8.

[22] Eifan O, Akkoc T, Yildiz A, Keles S, Ozdemir C, Bahceciler $\mathrm{NN}$ et al. Clinical efficacy and immunological mechanisms of sublingual and subcutaneous immunotherapy in asthmatic/rhinitis children sensitized to house dust mite: an open randomized controlled trial. Clinical \& Experimental Allergy 2010; 40:922-932.

[23] Frew AJ. Allergen immunotherapy. J Allergy Clin Immunol 2010 125: S306-313.

[24] Khinchi M. S, Poulse L. K, Carat F, André C, Hansen AB, Malling HJ. Clinical efficacy of sublingual and subcutaneous birch pollen allergen-specific immunotherapy: a randomized, placebo-controlled, double-blind, double-dummy study. Allergy 2004: 59: 45-53. 